\title{
Complete decomposable MS-algebras
}

\author{
Abd El-Mohsen Badawy ${ }^{1 *}$ and Ahmed Gaber ${ }^{2}$
}

\author{
*Correspondence: \\ abdel-mohsen.mohamed@science. \\ tanta.edu.eg \\ ${ }^{1}$ Department of Mathematics, \\ Faculty of Science, Tanta University, \\ Tanta, Egypt \\ Full list of author information is \\ available at the end of the article
}

\begin{abstract}
According to the characterization of decomposable MS-algebras in terms of triples $(M, D, \varphi)$, where $M$ is a de Morgan algebra, $D$ is a distributive lattice with 1 and $\varphi$ is a $(0,1)$-homomorphism of $M$ into $F(D)$, the filter lattice of $D$, we characterize complete decomposable MS-algebras in terms of complete decomposable MS-triples. Also, we describe the complete homomorphisms of complete decomposable MS-algebras by means of complete decomposable MS-triples.
\end{abstract}

Keywords: MS-algebras, Complete lattice, Complete decomposable MS-algebras, Complete decomposable MS-triples, Triple homomorphisms, Complete homomorphisms

AMS Mathematics Subject Classification (2010): Primary 06D30; Secondary 06D15.

\section{Introduction}

Morgan Stone algebras (or simply $M S$-algebras) are introduced and characterized by T.S. Blyth and J.C. Varlet [1] as a generalization of both de Morgan algebras and Stone algebras. In [2], T.S. Blyth and J.C. Varlet described the lattice $\Lambda$ (MS) of subclasses of the class MS of all $M S$-algebras. A. Badawy, D. Guffova, and M. Haviar [3] introduced and characterized decomposable $M S$-algebras by means of decomposable $M S$-triples. Moreover, they constructed a one-to-one correspondence between decomposable $M S$-algebras and decomposable $M S$-triples. A. Badawy and R. El-Fawal [4] studied many properties of decomposable $M S$-algebras in terms of decomposable $M S$-triples as homomorphisms and subalgebras. Also, they formulated and solved some fill in problems concerning homomorphisms and subalgebras of decomposable $M S$-algebras. A. Badawy [5] introduced the notion of $d_{L}$-filters of principal $M S$-algebras. Recently, A. Badawy [6] studied the relationship between de Morgan filters and congruences of decomposable $M S$-algebras. Also, many properties of ideals of $M S$-algebras are given in [7] and [8].

Several authors studied complete $p$-algebras, like C.C. Chain and G. Grätzer [9] for Stone algebras, S. El-Assar, and M. Atallah [10] for distributive $p$-algebras and P. Mederly [11] for modular $p$-algebras.

In this paper, we introduce complete decomposable $M S$-algebras and complete decomposable $M S$-triples. We show that a decomposable $M S$-algebra $L$ constructed from the decomposable $M S$-triple $(M, D, \varphi)$ is complete if and only if the triple $(M, D, \varphi)$ is complete. Also, a description of complete homomorphisms of decomposable $M S$-algebras is given in terms of complete decomposable $M S$-triples. 


\section{Preliminaries}

In this section, we present definitions and main results which are needed through this paper. We refer the reader to $[1-4,12-15]$ for more details.

A de Morgan algebra is an algebra $(L ; \vee, \wedge,-, 0,1)$ of type $(2,2,1,0,0)$ where $(L ; \vee, \wedge, 0,1)$ is a bounded distributive lattice and the unary operation of involution satisfies

$$
\overline{\bar{x}}=x, \overline{(x \vee y)}=\bar{x} \wedge \bar{y}, \overline{(x \wedge y)}=\bar{x} \vee \bar{y} \text {. }
$$

An MS-algebra is an algebra $\left(L ; \vee, \wedge,^{\circ}, 0,1\right)$ of type $(2,2,1,0,0)$ where $(L ; \vee, \wedge, 0,1)$ is a bounded distributive lattice and the unary operation ${ }^{\circ}$ satisfies

$x \leq x^{\circ \circ},(x \wedge y)^{\circ}=x^{\circ} \vee y^{\circ}, 1^{\circ}=0$.

The following Theorem gives the basic properties of $M S$-algebras.

Theorem 1 ([1, 12]). For any two elements $a, b$ of an MS-algebra $L$, we have

(1) $0^{\circ}=1$,

(2) $a \leq b \Rightarrow b^{\circ} \leq a^{\circ}$,

(3) $a^{000}=a^{\circ}$,

(4) $(a \vee b)^{\circ}=a^{\circ} \wedge b^{\circ}$,

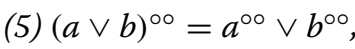

(6) $(a \wedge b)^{\circ \circ}=a^{\circ \circ} \wedge b^{\circ \circ}$.

Lemma 1 ([1,3]). Let L be an MS-algebra. Then

(1) $L^{\circ \circ}=\left\{x \in L: x=x^{\circ \circ}\right\}$ is a de Morgan subalgebra of $L$,

(2) $D(L)=\left\{x \in L: x^{\circ}=0\right\}$ is a filter (filter of dense elements) of $L$.

For any lattice $L$, let $F(L)$ denotes the set of all filters of $L$. It is known that, $(F(L) ; \wedge, \vee)$ is a distributive lattice if and only if $L$ is a distributive lattice, where the operation $\wedge$ and $\checkmark$ are given by

$F \wedge G=F \cap G$ and $F \vee G=\{x \in L: x \geq f \wedge g, f \in F, g \in G\}$, respectively for every $F, G \in$ $F(L)$.

Also, $[a)=\{x \in L: x \geq a\}$ is a principal filter of $L$ generated by $a$.

Definition 1 [9]. Let $L=\left(L ; \vee, \wedge, 0_{L}, 1_{L}\right)$ and $L_{1}=\left(L_{1} ; \vee, \wedge, 0_{L_{1}}, 1_{L_{1}}\right)$ be bounded lattices. The map $h: L \rightarrow L_{1}$ is called (0,1)-lattice homomorphism if

(1) $0_{L} h=0_{L_{1}}$ and $1_{L} h=1_{L_{1}}$,

(2) $h$ preserves joins, that is, $(x \vee y) h=x h \vee y h$ for every $x, y \in L$,

(3) $h$ preserves meets, that is, $(x \wedge y) h=x h \wedge y$ for every $x, y \in L$.

Definition 2 [14] A (0,1)-lattice homomorphism $h: L \rightarrow L_{1}$ of an MS-algebra $L$ into an MS-algebra $L_{1}$ is called a homomorphism if $x^{\circ} h=x h^{\circ}$ for all $x \in L$. If $L$ and $L_{1}$ are de Morgan algebras, then $h$ is called a de Morgan homomorphism.

Definition 3 [3] An MS-algebra $L$ is called decomposable MS-algebra iffor every $x \in L$ there exists $d \in D(L)$ such that $x=x^{\circ \circ} \wedge d$.

Definition 4 [3] A decomposable MS-triple is $(M, D, \varphi)$, where

(i) $(M ; \vee, \wedge,, 0,1)$ is a de Morgan algebra,

(ii) $(D ; \vee, \wedge, 1)$ is a distributive lattice with 1 , 
(iii) $\varphi$ is a $(0,1)$-homomorphism from $M$ into $F(D)$ such that for every element $a \in M$ and for every $y \in D$ there exists an element $t \in D$ with $a \varphi \cap[y)=[t)$.

Theorem 2 [3] (Construction Theorem) Let $(M, D, \varphi)$ be a decomposable MS-triple. Then

$$
L=\{(a, \bar{a} \varphi \vee[x)): a \in M, x \in D\}
$$

is a decomposable MS-algebra, if we define

$$
\begin{aligned}
& (a, \bar{a} \varphi \vee[x)) \vee(b, \bar{b} \varphi \vee[y))=(a \vee b, \overline{(a \vee b)} \varphi \vee[t)) \text { for somet } \in D, \\
& (a, \bar{a} \varphi \vee[x)) \wedge(b, \bar{b} \varphi \vee[y))=(a \wedge b, \overline{(a \wedge b)} \varphi \vee[x \wedge y)) \\
& (a, \bar{a} \varphi \vee[x))^{\circ}=(\bar{a}, a \varphi), \\
& 1_{L}=(1,[1)) \\
& 0_{L}=(0, D) .
\end{aligned}
$$

Conversely, every decomposable MS-algebra L can be associated with the decomposable MS-triple $\left(L^{\circ \circ}, D(L), \varphi(L)\right)$, where

$$
a \varphi(L)=\left[a^{\circ}\right)(L), a \in L^{\circ \circ} \text {. }
$$

The decomposable $M S$-algebra $L$ constructed in Theorem 2 is called the decomposable $M S$-algebra associated with the decomposable $M S$-triple $(M, D, \varphi)$ and the construction of $L$ described in Theorem 2 is called a decomposable $M S$-construction.

Corollary 1 [3] Let L be a decomposable MS-algebra associated with the decomposable MS-triple $(M, D, \varphi)$. Then

(1) $L^{\circ \circ}=\{(a, \bar{a} \varphi): a \in M\}$,

(2) $D(L)=\{(1,[x)): x \in D\}$,

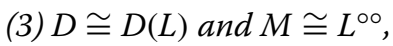

(4) The order of $L$ is given as follows: $(a, \bar{a} \varphi \vee[x)) \leq(b, \bar{b} \varphi \vee[y))$ iff $a \leq b$ and $\bar{a} \varphi \vee[x) \supseteq$ $\bar{b} \varphi \vee[y)$.

Definition 5 [14] $A$ lattice $L$ is called complete if $\inf _{L} H$ and $\sup _{L} H$ exist for each $\phi \neq$ $H \subseteq L$.

Definition 6 [14] A lattice $L$ is called conditionally complete if every upper bounded subset of $L$ has a supermum in $L$ and every lower bounded subset of $L$ has an infimum in $L$.

An $M S$-algebra $L$ is called complete if it is complete as a lattice.

Definition 7 [14] A lattice homomorphism $h: L \rightarrow L_{1}$ of a complete lattice $L$ into a complete lattice $L_{1}$ is called complete if

$\left(\inf _{L} H\right) h=\inf _{L_{1}} H h$ and $\left(\sup _{L} H\right) h=\sup _{L_{1}}$ Hh for each $\phi \neq H \subseteq L$.

A homomorphism $h: L \rightarrow L_{1}$ of a complete $M S$-algebra $L$ into a complete $M S$-algebra $L_{1}$ is called complete if it is complete as a lattice homomorphism. 


\section{Characterization of complete decomposable $M S$-algebras via triples}

In this section, we introduce and characterize complete decomposable $M S$-triples of complete decomposable $M S$-algebras.

Let $L$ be a decomposable $M S$-algebra $L$. For $\phi \neq N \subseteq L$, define $N^{\circ}$ as follows:

$N^{\circ}=\left\{n^{\circ}: n \in N\right\}$.

Lemma 2 If $L$ is a complete decomposable MS-algebra, then for $\phi \neq N \subseteq L, \phi \neq C \subseteq$ $L^{\circ \circ}$ and $\phi \neq E \subseteq D(L)$, we have

(1) $\left(\sup _{L} N\right)^{\circ}=\inf _{L} N^{\circ}$,

(2) $\sup _{L^{\circ \circ}} C=\left(\sup _{L} C\right)^{\circ \circ}=\left(\inf _{L} C^{\circ}\right)^{\circ}$,

(3) $\inf _{L^{\circ}} C=\inf _{L} C$,

(4) $\inf _{D(L)} E=\inf _{L} E$ and $\sup _{D(L)} E=\sup _{L} E$.

Proof (1). Let $x=\sup _{L} N$. Then $x \geq n$ for all $n \in N$ implies $x^{\circ} \leq n^{\circ}$. Hence $x^{\circ}$ is a lower bound of $N^{\circ}$. Let $y$ be a lower bound of $N^{\circ}$. Then $y \leq n^{\circ}$ for all $n \in N$ implies $y^{\circ} \geq n^{\circ \circ} \geq n$. So, $y^{\circ}$ is an upper bound of $N$. Thus $x \leq y^{\circ}$ as $x=\sup _{L} N$. This gives $x^{\circ} \geq y^{\circ \circ} \geq y$. Therefore $x^{\circ}=\inf _{L} N^{\circ}=\left(\sup _{L} N\right)^{\circ}$.

(2) Let $\sup _{L} C=x$. Then $x^{\circ \circ}=\left(\sup _{L} C\right)^{\circ \circ}$. We have to show that $x^{\circ \circ}=\sup _{L^{\circ \circ}} C$. Since $\sup _{L} C=x$, then $x \geq c$ for all $c \in C$. so, $x^{\circ \circ} \geq c^{\circ \circ}=c$ for all $c \in C$. Therefore $x^{\circ \circ}$ is an upper bound of $C$. Let $y$ be another upper bound of $C$ in $L^{\circ \circ}$. Then $y \geq c$ for all $c \in C$. Thus $y^{\circ \circ} \geq c^{\circ \circ}=c$. Hence $y^{\circ \circ}$ is an upper bound of $C$. So $y^{\circ \circ} \geq x$ as $x=\sup _{L} C$. It follows that $y=y^{\circ \circ} \geq x^{\circ \circ}$. Hence $x^{\circ \circ}$ is the least upper bound of $C$. Since $x^{\circ \circ} \in L^{\circ \circ}$, then $x^{\circ \circ}=\sup _{L^{\circ \circ}} C$. By $(1)$ we have $\left(\sup _{L} C\right)^{\circ \circ}=\left(\inf _{L} C^{\circ}\right)^{\circ}$.

(3) Let $x=\inf _{L} C$. Then $x \leq c$ for all $c \in C$. Then $x^{\circ \circ} \leq c^{\circ \circ}=c$. Hence $x^{\circ \circ}$ is a lower bound of $C$. Thus $x \geq x^{\circ \circ}$ as $x=\inf _{L} C$. But $x \leq x^{\circ \circ}$. Then $x^{\circ \circ}=x$ and $x \in L^{\circ \circ}$. Thus $\inf _{L^{\circ \circ}} C=x$.

(4) Let $x=\inf _{L} E$ and $y=\inf _{D(L)} E$. Then $x \leq e$ and $y \leq e$ for all $e \in E$ imply that $x=y$. Now we prove $\sup _{D(L)} E=\sup _{L} E$. Let $y=\sup _{L} E$. Then $y \geq e$ for all $e \in E$. It follows that $y^{\circ} \leq e^{\circ}=0$. Then $y \in D(L)$ implies $y=\sup _{D(L)} E$.

Let $(M, D, \varphi)$ be a decomposable $M S$-triple. For any $\emptyset \neq E \subseteq D$, consider the set $M_{E}$ as follows:

$M_{E}=\{a \in M: \bar{a} \varphi \vee[z) \supset E$ for some $z \in D\}$.

Lemma 3 Let $(M, D, \varphi)$ be a decomposable MS-triple. For any $\emptyset \neq E \subseteq D$, we have

(1) $M_{E}$ is an ideal of $M$,

(2) $[E)=\cup\{[t): t \in E\}$,

(2) $M_{E}=M_{[E)}$.

Proof (1). Let $a, b \in M_{E}$. Then $\bar{a} \varphi \vee\left[z_{1}\right) \supset E$ and $\bar{b} \varphi \vee\left[z_{2}\right) \supset E$ for some $z_{1}, z_{2} \in D$. Hence $E \subset\left(\bar{a} \varphi \vee\left[z_{1}\right)\right) \cap\left(\bar{b} \varphi \vee\left[z_{2}\right)\right)=\overline{(a \vee b)} \varphi \vee[t)$ for some $t \in D$ (see Theorem 2). It follows that $a \vee b \in M_{E}$. Now, let $a \in M_{E}$ and $c \in M$. Then, $\exists z \in D$ such that $\bar{a} \varphi \vee[z) \supset E$. Since $a \wedge c \leq a$, then $\overline{a \wedge c} \geq \bar{a}$. This gives $\overline{(a \wedge c)} \varphi \supseteq \bar{a} \varphi$. It follows that $\overline{(a \wedge c)} \varphi \vee[z) \supseteq \bar{a} \varphi \vee[z) \supset E$. Then $a \wedge c \in M_{E}$. Consequently, $M_{E}$ is an ideal of $M$.

(2) Obvious. 
(3) Clearly, $M_{[E]} \subseteq M_{E}$. Let $a \in M_{E}$. Then, $\exists z \in D$ such that $\bar{a} \varphi \vee[z) \supset E$. Since $\bar{a} \varphi \vee[z)$ is a filter of $D$ and $[E]$ is the smallest filter of $D$ containing $E$, then $\bar{a} \varphi \vee[z) \supset[E$ ). Hence, $a \in M_{[E)}$ and $M_{E} \subseteq M_{[E)}$. Therefore, $M_{E}=M_{[E)}$.

Definition 8 A complete decomposable MS-triple is a decomposable MS-triple $(M, D, \varphi)$ satisfying the following conditions:

(i) $M$ is complete,

(ii) $D$ is conditionally complete,

(iii) For each $\emptyset \neq E \subseteq D$, the set $M_{E}$ has the greatest element in $M$.

Theorem 3 Let L be a complete decomposable MS-algebra constructed from the decomposable MS-triple $(M, D, \varphi)$. Then, the triple $(M, D, \varphi)$ is complete.

Proof Since $L$ is associated with the decomposable $M S$-triple $(M, D, \varphi)$, then by Theorem 2, we have

$L=\{(a, \bar{a} \varphi \vee[x)): a \in M, x \in D\}$.

Corollary 1(1)-(3), gives

$L^{\circ \circ}=\{(a, \bar{a} \varphi): a \in M\} \cong M$ and $D(L)=\{(1,[x)): x \in D\} \cong D$.

We have to prove that a decomposable $M S$-triple $(M, D, \varphi)$ is complete. So we proceed to prove (i)-(iii) of Definition 8. For (i), let $\emptyset \neq C \subseteq M$. Consider a subset $\dot{C}=\{(c, \bar{c} \varphi)$ : $c \in C\}$ of $L^{\circ \circ}$ corresponding to $C$. Since $L$ is complete, then $\inf _{L} \dot{C}=(a, \bar{a} \varphi \vee[x))$ for some $(a, \bar{a} \varphi \vee[x)) \in L$. Thus, $(a, \bar{a} \varphi \vee[x)) \leq(c, c \varphi)$ for all $c \in C$. Then $a \leq c$ for all $c \in C$ implies that $a$ is a lower bound of $C$. We verify that $a$ is the greatest lower bound of $C$ in $M$. Let $b$ be a lower bound of $C$. Then $b \leq c$ for all $c \in C$. This gives $\bar{b} \varphi \supseteq \bar{c} \varphi$. Therefore, $(b, \bar{b} \varphi) \leq$

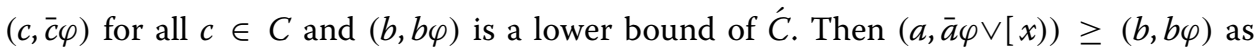
$\inf _{L} C=(a, \bar{a} \varphi \vee[x))$. Consequently, $a \geq b$ and $a=\inf _{M} C$. Since $a=\inf _{M} C$ and $M$ is bounded above by 1 , then, $M$ is complete.

Now we prove (ii). Let $\phi \neq E \subseteq D$. Consider $E \subseteq D(L)$ corresponding to $E$. Then $\dot{E}=\{(1,[e)): e \in D\}$.

Let $z$ be a lower bound of $E$. Since $L$ is complete, then $\inf _{L} \dot{E}$ exists. Let $\inf _{L} E=$ $(a, \bar{a} \varphi \vee[x))$. Since $z \leq e$ for all $e \in E$ as $z$ is a lower bound of $E$. Then, $[z) \supseteq[e)$ and $(1,[z)) \leq(1,[e))$. Thus, $(1, z)$ is a lower bound of $E$. Then, $(a, \bar{a} \varphi \vee[x)) \geq(1,[z))$ because of $\inf _{L}{ }^{\prime} E=(a, \bar{a} \varphi \vee[x))$. This implies that $a \geq 1$ and $\bar{a} \varphi \vee[x) \subseteq[z)$. Consequently, $a=1$ and $\bar{a} \varphi \vee[x]=0 \varphi \vee[x]=[x)$. Thus $[x] \subseteq[z)$ implies $x \geq z$. This shows that $x$ is the greatest lower bound of $E$ in $D$ and $x=\inf _{D} E$. Using a similar way, we can show that, if $E$ has an upper bound, then $\sup _{D} E$ exists. Therefore, $D$ is a conditionally complete lattice as required.

Now we prove (iii). Let $\emptyset \neq E \subseteq D$. Consider $E \subseteq D(L)$ corresponding to $E$. Then

$$
\dot{E}=\{(1,[x)): x \in E\}
$$

Since $L$ is complete, then $\inf _{L} E^{\prime}$ exists. Let $(b, \bar{b} \varphi \vee[z))=\inf _{L} E^{\prime}$. We show that $b$ is the largest element of $M_{E}$. Since $(b, \bar{b} \varphi \vee[z))=\inf _{L} E$, then $(b, \bar{b} \varphi \vee[z)) \leq(1,[x)), \forall x \in E$. This gives $b \leq 1$ and $\bar{b} \varphi \vee[z) \supseteq[x), \forall x \in E$. Therefore, $\bar{b} \varphi \vee[z) \supseteq \cup_{x \in E}[x]=[E) \supset E$. Thus, $b \in M_{E}$. Now, let $c \in M_{E}$. Then $\bar{c} \varphi \vee[y) \supset E$ for some $y \in D$. It follows that $\bar{c} \varphi \vee[y) \supseteq[E) \supseteq[x)$ for all $x \in E$. Hence, $(1,[x)) \leq(c, \bar{c} \varphi \vee[y))$ for all $x \in E$. Thus, $(c, \bar{c} \varphi \vee[y))$ is a lower bound of $E$ and therefore $(c, \bar{c} \varphi \vee[y)) \leq(b, \bar{b} \varphi \vee[z))$. Then, $c \leq b$. 
This deduce that $b$ is the largest element of $M_{E}$ in $M$. Therefore, $(M, D, \varphi)$ is a complete decomposable $M S$-triple.

The converse of the above theorem is given in the following.

Theorem 4 Let L be a decomposable MS-algebra constructed from the complete decomposable MS-triple $(M, D, \varphi)$. Then L is complete.

Proof Let $(M, D, \varphi)$ be a complete decomposable $M S$-triple. Then -(iii) of Definition 8 hold. Let $\emptyset \neq N \subseteq L$, where $L$ is constructed as in construction Theorem from the decomposable $M S$-triple $(M, D, \varphi)$ as follows:

$L=\{(a, \bar{a} \varphi \vee[x)): a \in M, x \in D\}$.

Since $L$ is bounded, it is enough to show the existence of $\inf _{L} N$. Denote $a=\inf _{M} N^{\circ \circ}$ and $F=\cup\{[t):(c, \bar{c} \varphi \vee[t)) \in N$ for some $c \in M\}(\cup$ means the union in $F(D))$. Let $b=$ $\max M_{F}$. Now, we prove that there exists an element $z \in D$ such that $\bar{b} \varphi \vee[z) \supset F$ and if $\bar{b} \varphi \vee[y) \supset F$ for some $y \in D$ then $\bar{b} \varphi \vee[y] \supseteq \bar{b} \varphi \vee[z)$. For this purpose, consider the following set:

$\left\{x_{\gamma}: \gamma \in \Gamma\right.$ for all $x_{\gamma}$ with $\left.\bar{b} \varphi \vee\left[x_{\gamma}\right) \supset F\right\}$.

Thus, we have to find $\mathrm{a} z \in D$ with $\bar{b} \varphi \vee[y) \supset F$ and $\bar{b} \varphi \vee[y] \supseteq \bar{b} \varphi \vee[z)$ for all $\gamma \in \Gamma$. The set $\left\{x_{\gamma}: \gamma \in \Gamma\right.$ for all $x_{\gamma}$ with $\left.\bar{b} \varphi \vee\left[x_{\gamma}\right) \supset F\right\}$ is bounded from above. Then, by (ii), there exists $s=\sup _{D}\left\{x_{\gamma}: \gamma \in \Gamma\right\}$. We prove that $\cap_{\gamma \in \Gamma}\left[x_{\gamma}\right)=[s)$.

$$
\begin{aligned}
y \in \cap_{\gamma \in \Gamma}\left[x_{\gamma}\right) & \Leftrightarrow y \in\left[x_{\gamma}\right), \forall \gamma \in \Gamma \\
& \Leftrightarrow y \geq x_{\gamma}, \forall \gamma \in \Gamma \\
& \Leftrightarrow y \text { is an upper bound of }\left\{x_{\gamma}: \gamma \in \Gamma\right\} \\
& \Leftrightarrow y \geq s \text { as } s=\sup _{D}\left\{x_{\gamma}: \gamma \in \Gamma\right\} \\
& \Leftrightarrow y \in[s) .
\end{aligned}
$$

Then it is sufficient to prove the following equality.

$$
\cap_{\gamma \in \Gamma}\left(\bar{b} \varphi \vee\left[x_{\gamma}\right)\right)=\bar{b} \varphi \vee \cap_{\gamma \in \Gamma}\left[x_{\gamma}\right)=\bar{b} \varphi \vee[s)
$$

Let $t \in \bar{b} \varphi \vee[s)$. Then

$$
\begin{aligned}
t \in \bar{b} \varphi \vee[s) & \Rightarrow t \geq t_{1} \wedge s \text { where } t_{1} \in \bar{b} \varphi \\
& \Rightarrow t \geq t_{1} \wedge\left(s \vee x_{\gamma}\right) \text { as } s \geq x_{\gamma} \text { for all } \gamma \in \Gamma \\
& \Rightarrow t \geq\left(t_{1} \wedge s\right) \vee\left(t_{1} \wedge x_{\gamma}\right) \\
& \Rightarrow t \geq t_{1} \wedge x_{\gamma} \\
& \Rightarrow t \in \bar{b} \varphi \vee\left[x_{\gamma}\right) \text { for all } \gamma \in \Gamma .
\end{aligned}
$$

Then $\bar{b} \varphi \vee \cap_{\gamma \in \Gamma}\left[x_{\gamma}\right) \subseteq \bar{b} \varphi \vee\left[x_{\gamma}\right)$ implies $\bar{b} \varphi \vee \cap_{\gamma \in \Gamma}\left[x_{\gamma}\right) \subseteq \cap_{\gamma \in \Gamma}\left(\bar{b} \varphi \vee\left[x_{\gamma}\right)\right)$. Conversely, let $y \in \cap_{\gamma \in \Gamma}\left(\bar{b} \varphi \vee\left[x_{\gamma}\right)\right)$. Then $y \in \bar{b} \varphi \vee\left[x_{\gamma}\right)$ for all $\gamma \in \Gamma$. Hence $y \geq t \wedge z$ for $t \in \bar{b} \varphi$ and $z \in\left[x_{\gamma}\right)$ for all $\gamma \in \Gamma$. It follows that $z \geq x_{\gamma}$ for all $\gamma \in \Gamma$. This means that $z$ is an upper bound of the set $\left\{x_{\gamma}: \gamma \in \Gamma\right\}$. Then $s \leq z$ as $s=\sup _{D}\left\{x_{\gamma}: \gamma \in \Gamma\right\}$. Now 


$$
\begin{aligned}
y & \geq t \wedge z \\
& =t \wedge(s \vee z) \text { as } s \leq z \\
& =(t \wedge s) \vee(t \wedge z) \text { by distributivity of } D \\
& \geq t \wedge s \in \bar{b} \varphi \vee[s) .
\end{aligned}
$$

Then $y \in \bar{b} \varphi \vee[s)$. Therefore, $\cap_{\gamma \in \Gamma}\left(\bar{b} \varphi \vee\left[x_{\gamma}\right)\right) \subseteq \bar{b} \varphi \vee[s)$.

We prove the existence of $\inf _{L} N$. First, we claim that $i=(a \wedge b, \overline{(a \wedge b)} \varphi \vee[z))=\inf _{L} N($ we put then $z=s)$.

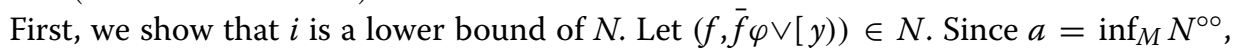
we get $a \leq f$. So, $a \wedge b \leq a \leq f$. Then $a \wedge b \leq f$ implies that $\overline{a \wedge b} \geq \bar{f}$. Consequently, $\overline{(a \wedge b)} \varphi=\bar{a} \varphi \vee \bar{b} \varphi \supseteq \bar{f} \varphi$. Moreover, $[y) \subseteq F \subseteq \bar{b} \varphi \vee[z)$ as $y \in F$. Then

$$
\begin{aligned}
\overline{(a \wedge b)} \varphi \vee[z) & =(\bar{a} \vee \bar{b}) \varphi \vee[z) \\
& =(\bar{a} \varphi \vee \bar{b} \varphi) \vee(\bar{b} \varphi \vee[z)) \\
& \supseteq \bar{f} \varphi \vee[y) .
\end{aligned}
$$

Then $(a \wedge b, \overline{(a \wedge b)} \varphi \vee[z)) \leq(f, \bar{f} \vee[y))$ for all $(f, \bar{f} \vee[y)) \in N$. Therefore, $i$ is a lower bound of $N$. It remains to show that $i$ is the greatest lower bound of $N$. Let $(c, \bar{c} \varphi \vee[x))$ be a lower bound of $N$. Then, $(c, \bar{c} \varphi \vee[x)) \leq(f, \bar{f} \varphi \vee[y)), \forall(f, \bar{f} \varphi \vee[y)) \in N$. So, $c \leq f, \forall f \in$ $N^{\circ \circ}$. Then $c$ is a lower bound of $N^{\circ \circ}$. Thus $c \leq a$ as $a=\inf _{M} N^{\circ \circ}$. On the other hand, $\bar{c} \varphi \vee[x) \supseteq \bar{f} \varphi \vee[y), \forall(f, \bar{f} \varphi \vee[y)) \in N$. So, $\bar{c} \varphi \vee[x) \supseteq[y), \forall y \in F$. Therefore, $\bar{c} \varphi \vee[x] \supseteq F$. Hence, $\bar{c} \varphi \vee[x] \supseteq \bar{b} \varphi \vee[z)$ by using equality (1). Then $\bar{c} \varphi \vee[x] \supseteq F$ implies that $c \in M_{F}$. So, $c \leq b$ as $b=\max _{M} M_{F} \in M$. Now, we have $c \leq a$ and $c \leq b$. Then $c \leq a \wedge b$. Moreover, we have $\bar{c} \varphi \supseteq \bar{a} \varphi$ because of $c \leq a$. Also, $\bar{c} \varphi \vee[x) \supseteq \bar{b} \varphi \vee[z)$. So, $\bar{c} \varphi \vee[x) \supseteq \bar{a} \varphi \vee \bar{b} \varphi \vee[z]=$ $\overline{(a \wedge b)} \varphi \vee[z)$. Therefore, $(c, \bar{c} \varphi \vee[x)) \leq i$. Then $i=\inf _{L} N$ and $L$ is complete.

Corollary 2 If $M$ and $D$ are complete, then so is $L$.

Proof. We need only to prove that the condition (iii) of Definition 8 holds. Let $E \subseteq D$ and $t=\inf _{D} E$. Then, $[t)=\left[\inf _{D} E\right) \supseteq E$. So, $(1, \overline{1} \varphi \vee[t))=(1,[t)) \in L$. Therefore, $1 \in M_{E}$. Hence, by the above Theorem, $L$ is complete.

Corollary 3 If $M$ is finite and $D$ is conditionally complete, then $L$ is complete.

Proof Since $M$ is finite and $M_{E}$ is an ideal of $M$ (see Lemma 1(1)), then $M$ is complete and $M_{E}$ is a principal ideal of $M$. Therefore, $M_{E}$ contains the greatest element in $M$. So, the conditions (i)-(iii) of Definition 8 are satisfied and consequently, $L$ is complete.

Combining Theorems 3 and 4, we get the following theorem.

Theorem 5 Let $L$ be a decomposable MS-algebra constructed from the decomposable MS-triple $(M, D, \varphi)$. Then $L$ is complete if and only if $(M, D, \varphi)$ is complete.

Let $L$ be a complete decomposable $M S$-algebra. In the proof of Theorem 4 arbitrary meets in $L$ are described. In the following Lemma, we describe joins in $L$. 
Lemma 4 Let $L$ be a complete decomposable MS-algebra constructed from the decomposable MS-triple $(M, D, \varphi)$. Let $\phi \neq N \subseteq L$ and $a=\sup _{M} N^{\circ \circ}$. Then there exists an element $z \in D$ such that $[z)=\bigcap\{\bar{c} \varphi \vee[t):(c, \bar{c} \varphi \vee[t)) \in N\} \cap a \varphi$ and $\sup N=$ $(a, \bar{a} \varphi \vee[z))$.

Proof Let $\phi \neq N \subseteq L$ and $\sup _{L} N=(b, \bar{b} \varphi \vee[z))$. We can assume that $z \in a \varphi$. We prove that $b=a=\sup _{M} N^{\circ \circ}$. Using Lemma 2(2), we get

$$
\sup _{M} N^{\circ \circ}=\left(\sup _{L} N\right)^{\circ \circ}=(b, \bar{b} \varphi \vee[z))^{\circ \circ}=(b, \bar{b} \varphi) .
$$

But $a=(a, \bar{a} \varphi)=\sup _{M} N^{\circ \circ}$. Then $b=a$. Hence, $\bar{a} \varphi \vee[z)$ is the greatest filter of the form $\bar{a} \varphi \vee[x), x \in D$ with

$\bar{a} \varphi \vee[z)) \subset \bar{c} \varphi \vee[t)$ for each $(c, \bar{c} \varphi \vee[t)) \in N$.

The last condition is equivalent to

$[z) \subset \bigcap\{\bar{c} \varphi \vee[t):(c, \bar{c} \varphi \vee[t)) \in N\} \cap a \varphi$.

Let $\bigcap\{\bar{c} \varphi \vee[t):(c, \bar{c} \varphi \vee[t)) \in N\} \cap a \varphi=R$. If $[z) \neq R$, then there is $y \in R, y \nsucceq z$. It follows that $y \wedge z<z$ and $y \wedge z \in R$. Then $[z) \subset[y \wedge z)$ implies $\bar{a} \varphi \vee[z) \subset \bar{a} \varphi \vee[y \wedge z)$. Since $y \wedge z \in R$ then $[y \wedge z) \subset \bar{c} \varphi \vee[t)$ for all $(c, \bar{c} \varphi \vee[t)) \in N$. Since $a \geq c\left(\operatorname{as} a=\sup _{M} N^{\circ \circ}\right)$ then $\bar{a} \leq \bar{c}$. It follows that $\bar{a} \varphi \leq \bar{c} \varphi$. Therefore, $\bar{a} \varphi \vee[y \wedge z) \subset \bar{c} \varphi \vee[t)$ for all $(c, \bar{c} \varphi \vee[t)) \in N$. Consequently,

$\bar{a} \varphi \vee[z) \subset \bar{a} \varphi \vee[y \wedge z) \subset \bar{c} \varphi \vee[t)$ for all $(c, \bar{c} \varphi \vee[t)) \in N$,

which contradicts the maximality of $\bar{a} \varphi \vee[z)$.

\section{Complete homomorphisms via complete triple homomorphisms}

In this section, we introduce complete triple homomorphisms of complete decomposable $M S$-algebras. Then, we characterize complete homomorphisms of complete decomposable $M S$-algebras in terms of complete triple homomorphisms. For this purpose, we recall from [4], the notion of triple homomorphism of decomposable $M S$-triples and related properties which will be used in rest of the paper.

Definition 9 [4] Let $(M, D, \varphi)$ and $\left(M_{1}, D_{1}, \varphi_{1}\right)$ be decomposable MS-triples. A triple homomorphism of the triple $(M, D, \varphi)$ into $\left(M_{1}, D_{1}, \varphi_{1}\right)$ is a pair $(f, g)$, where $f$ is a homomorphism of $M$ into $M_{1}, g$ is a homomorphism of $D$ into $D_{1}$ preserving 1 such that for every $a \in M$,

$$
a \varphi g \subseteq \operatorname{af} \varphi_{1}
$$

Lemma 5 [4] Let $(f, g)$ be a triple homomorphism of a decomposable MS-triple $(M, D, \varphi)$ into a decomposable MS-triple $\left(M_{1}, D_{1}, \varphi_{1}\right)$. Let $a, b \in M$ and $x, y, t \in D$. Then

(i) $a \varphi \cap[y)=\left[t\right.$ implies af $\varphi_{1} \cap[y g)=[t g)$,

(ii) $\left(\bar{a} f \varphi_{1} \vee[x g)\right) \cap\left(\bar{b} f \varphi_{1} \vee[y g)\right)=\overline{(a \vee b)} f \varphi_{1} \vee[t g)$.

Theorem 6 [4] Let $L$ and $L_{1}$ be decomposable MS-algebras, $(M, D, \varphi)$ and $\left(M_{1}, D_{1}, \varphi_{1}\right)$ be the associated decomposable MS-triples, respectively. Let $h$ be a homomorphism of $L$ into $L_{1}$ and $h_{M}, h_{D}$ the restrictions of $h$ to $M$ and $D$, respectively. Then $\left(h_{M}, h_{D}\right)$ is a triple homomorphism of the decomposable MS-triples. Conversely, every triple homomorphism $(f, g)$ of the decomposable MS-triples uniquely determines a homomorphism $h$ of $L$ into $L_{1}$ with $h_{M}=f, h_{D}=g$ by the following rule: 
$x h=x^{\circ \circ} f \wedge d g$, for all $x \in L$,

where $x=x^{\circ \circ} \wedge d$ for some $d \in D(L)$.

If $L$ and $L_{1}$ are represented as in the construction Theorem then (3) reads

$$
(a, \bar{a} \varphi \vee[x)) h=(a f, \overline{(a f)} \varphi \vee[x g)) \text { for all }(a, \bar{a} \varphi \vee[x)) \in L .
$$

In the following, we will write $L=(M, D, \varphi)$ to indicate that $(M, D, \varphi)$ is the decomposable $M S$-triple associated with $L$, that is, $L^{\circ \circ}=M, D(L)=D$, and $\varphi(L)=\varphi$. Let $L=(M, D, \varphi)$ and $L_{1}=\left(M_{1}, D_{1}, \varphi_{1}\right)$ be decomposable $M S$-algebras, we will write $h=(f, g)$ to indicate that $(f, g):(M, D, \varphi) \rightarrow\left(M_{1}, D_{1}, \varphi_{1}\right)$ is the triple homomorphism of decomposable $M S$-triples corresponding to the homomorphism $h$ of $L$ into $L_{1}$.

Lemma 6 Let $h=(f, g)$ be a homomorphism of a decomposable MS-algebra L onto a decomposable MS-algebra $L_{1}$. Then for each $a \in L^{\circ \circ}$, we have

$a \varphi g=a f \varphi_{1}$.

Proof We have, $a \varphi g \subseteq a f \varphi_{1}$ by (2). It remains to show that $a f \varphi_{1} \subseteq a \varphi g$. Let $y \in a f \varphi_{1}$. Then

$$
y \in\left[(a f)^{\circ}\right) \cap D\left(L_{1}\right)=\left[(a h)^{\circ}\right) \cap D\left(L_{1}\right) \text { implies } y \in\left[(a h)^{\circ}\right) \text { and } y \in D\left(L_{1}\right) .
$$

Then $y \geq(a h)^{\circ}=a^{\circ} h$. Since $h$ is onto, then $g: D(L) \rightarrow D\left(L_{1}\right)$ is also onto. Hence, there exists $x \in D(L)$ such that $x h=y$. Evidently, $a^{\circ} \vee x \in\left[a^{\circ}\right) \cap D(L)$ and

$\left(a^{\circ} \vee x\right) h=a^{\circ} h \vee x h=x h$ as $x h=y \geq a^{\circ} h$.

Therefore, $y \in\left[a^{\circ} h\right) \cap D\left(L_{1}\right)=\left(\left[a^{\circ}\right) h \cap D g\right)=\left(\left[a^{\circ}\right) \cap D\right) g=a \varphi g$.

Now, we introduce the concept of complete triple homomorphism.

Definition 10 A triple homomorphism ( $f, g)$ of a decomposable MS-triple $(M, D, \varphi)$ into a decomposable MS-triple $\left(M_{1}, D_{1}, \varphi_{1}\right)$ is called complete if the following conditions are satisfied

(i) fis a complete homomorphism of $M$ and $M_{1}$,

(ii) $g$ is a complete homomorphism of $D$ and $D_{1}$,

(iii) $\left(\max M_{E}\right) f=\max M_{1 E g}$ for each $\phi \neq E \subseteq D$.

Remark 1 First, we observe that the map $g: D \rightarrow D_{1}$ is a complete means that $\left(\sup _{D} E\right) g=\sup _{D_{1}} E g$ for any $E \subseteq D$ and if $\inf _{D} E$ and $\inf _{D_{1}} M g$ exist then $\left(\inf _{D} E\right) g=$ $\inf _{D_{1}} E g$.

Theorem 7 Let $L=(M, D, \varphi)$ and $L_{1}=\left(M_{1}, D_{1}, \varphi_{1}\right)$ be complete decomposable MSalgebras and let $h=(f, g)$ be a homomorphism of $L$ onto $L_{1}$. Then $h$ is complete if and only if $(f, g)$ is complete.

Proof The decomposable $M S$-triples $(M, D, \varphi)$ and $\left(M_{1}, D_{1}, \varphi_{1}\right)$ are associated with $L$ and $L_{1}$, respectively. Let $h=(f, g)$ be a complete homomorphism of $L$ onto $L_{1}$. Then $f$ is 
a de Morgan homomorphism of $M$ onto $M_{1}$ and $g$ is a lattice homomorphism of $D$ onto $D_{1}$ preserving 1 . We have to verify that $f$ and $g$ are complete. Let $\phi \neq N \subseteq M$. Then

$$
\begin{array}{r}
\left(\inf _{M} N\right) f=\left(\inf _{L} N\right) f=\left(\inf _{L} N\right) h=\inf _{L_{1}} N h=\inf _{L_{1}} N f=\inf _{M_{1}} N f \text { by Lemma 2(3), } \\
\left(\sup _{M} N\right) f=\left(\sup _{L} N\right)^{\circ \circ} f=\left(\left(\sup _{L} N\right) h\right)^{\circ}=\left(\sup _{L_{1}} N h\right)^{\circ}=\sup _{M_{1}} N f \text { by Lemma 2(2). }
\end{array}
$$

Thus, $f$ is complete. We prove that $g$ is complete. Let $\phi \neq E \subseteq D$. Then

$\left(\sup _{D} E\right) g=\left(\sup _{L} E\right) g=\left(\sup _{L} N\right) h=\sup _{L_{1}} N h=\sup _{D_{1}} E g$ by Lemma 2(4).

If $\inf _{D} E$ and $\inf _{D_{1}} E g$ exist, then

$\left(\inf _{D} E\right) g=\left(\inf _{L} E\right) g=\left(\inf _{L} N\right) h=\inf _{L_{1}} N h=\inf _{D_{1}} E g$ by Lemma 2(4).

Now, we prove (iii). Let $\phi \neq E \subseteq D$. Consider $E$ corresponding the set $E$ on $D(L)$, where $\hat{E}=\{(1,[x)): x \in E\} \subseteq D(L)$.

By (4), we have

$\hat{E} h=\{(1,[x g)): x \in E\} \subseteq D\left(L_{1}\right)$.

Since $h$ is complete, then $\left(\inf _{L} E\right) h=\inf _{L_{1}} E h$ for each $\phi \neq E \subseteq L$. Hence, $\left(\inf _{L} E\right)^{\circ \circ}=$ $\max M_{E}$ (see the proof of Theorem 3 ) and similarly $\left(\inf _{L_{1}} E h\right)^{\circ \circ}=\max M_{1 E g}$. Conversely, assume that (i)-(iii) hold and let $h=(f, g)$ be a homomorphism of $L$ onto $L_{1}$. We have to show that $h$ is complete. First we prove that for $\phi \neq H \subseteq L$, $\left(\inf _{L} H\right) h=\inf _{L_{1}} H h$ holds. Consider $E=\bigcup\{[t):(c, \bar{c} \varphi \vee[x)) \in M\}$. Let $\max M_{E}=b$ and $\inf _{M} H^{\circ \circ}=a$. Then according to the proof of Theorem 4, we get

$$
i=(a \wedge b, \overline{(a \wedge b)} \varphi \vee[z))=\inf _{L} H \text {, where } z=\sup _{D}\left\{x_{\gamma}: \bar{b} \varphi \vee\left[x_{\gamma}\right) \supset E\right\} \text {. Using (4), }
$$

we have

$$
H h=\{(c f, \bar{c} f \varphi \vee[x g)):(c, \bar{c} \varphi \vee[x)) \in H\}
$$

and

$$
i h=((a \wedge b) f, \overline{(a \wedge b) f} \varphi \vee[z g))=\left(\inf _{L} H\right) h .
$$

Now, $\inf _{L_{1}}(H f)^{\circ \circ}=\left(\inf _{M} H^{\circ \circ}\right) f=a f$ by (i) and $\max M_{1 E g}=\left(\max M_{E}\right) f=b f$ by (iii).

Since $L_{1}$ is complete and $H h \subset L_{1}$ then again according to the proof of Theorem 4, we get $\inf _{L_{1}} H h=\left((a \wedge b) f, \overline{(a \wedge b) f} \varphi \vee\left[z_{1}\right)\right)=i h$, where $z_{1}=\sup \left\{x_{\gamma} g: \gamma \in \Gamma\right\}=\left(\sup \left\{x_{\gamma}:\right.\right.$ $\gamma \in \Gamma\}) g=z g$ as $g$ is an onto homomorphism. Therefore, $\inf _{L} M h=\left(\inf _{L_{1}} M\right) h$.

Now, we prove that $\left(\sup _{L} H\right) h=\sup _{L_{1}} H h$. By Lemma 4, $\sup _{L}(M)=(a, \bar{a} \varphi \vee[z))$, where $a=\sup _{M} H^{\circ \circ}$ and $[z)=\bigcap\{\bar{c} \varphi \vee[t):(c, \bar{c} \varphi \vee[t)) \in H\} \cap a \varphi$. Then $\sup _{L_{1}} H h=$ $\left(a_{1}, \bar{a}_{1} \varphi_{1} \vee\left[z_{1}\right)\right)$, where $a_{1}=\sup _{M_{1}}(H h)^{\circ \circ}=\sup _{L_{1}}(H h)^{\circ \circ}=\sup _{L_{1}} H^{\circ \circ} h=$ $\left(\sup _{L} M^{\circ \circ}\right) h=\left(\sup _{M} H^{\circ \circ}\right) h=a h=a f$ (by using Lemma 2(2) and (i) of Definition 9) and $\left[z_{1}\right)=\bigcap\left\{\bar{c} f \varphi_{1} \vee[t g):(c, \bar{c} \varphi \vee[t)) \in H\right\} \cap a_{1} \varphi_{1}$. We show that $z g=z_{1}$. We have $c f \varphi_{1}=c \varphi g$ by Lemma 6 and $\bar{c} \varphi g \vee[t g)=(\bar{c} \varphi \vee[t)) g$ by Lemma $5(1)$. Then

$$
\begin{aligned}
{\left[z_{1}\right) } & =\bigcap\{\bar{c} \varphi \vee[t)) g:(c, \bar{c} \varphi \vee[t)) \in H\} \cap a \varphi g \\
& =(\bigcap\{\bar{c} \varphi \vee[t):(c, \bar{c} \varphi \vee[t)) \in H\} \cap a \varphi) g \\
& =[z g)
\end{aligned}
$$

which implies $z_{1}=z g$. Therefore, $\left(\sup _{L} H\right) h=\sup _{L_{1}} H h$ and $h$ is complete. 


\section{Authors' contributions}

The manuscript is being submitted by me (Corresponding author) on behalf of all the authors. The manuscript is the original work of all authors. All authors made a significant contribution to this study. All authors have read and approved the final version of the manuscript.

\section{Funding}

No fund.

\section{Availability of data and materials}

Not applicable.

\section{Competing interests}

The authors declare that they have no competing interests.

\section{Publisher's Note}

Springer Nature remains neutral with regard to jurisdictional claims in published maps and institutional affiliations.

\section{Author details}

${ }^{1}$ Department of Mathematics, Faculty of Science, Tanta University, Tanta, Egypt. ${ }^{2}$ Department of Mathematics, Faculty of Science, Ain Shams University, Cairo, Egypt.

Received: 29 December 2018 Accepted: 26 March 2019

Published online: 01 August 2019

\section{References}

1. Blyth, T. S., Varlet, J. C.: On a common abstraction of de Morgan algebras and Stone algebras. Proc. Roy. Soc. Edinburgh. 94A, 301-308 (1983)

2. Blyth, T. S., Varlet, J. C.: Subvarieties of the class of $M S$-algebras. Proc. Roy. Soc. Edinburgh. 95A, 157-169 (1983)

3. Badawy, A., Guffova, D., Haviar, M.: Triple construction of decomposable $M S$-algebras. Acta Univ. Palacki. Olomuc. Fac. Rer. Nat. Math. 51(2), 53-65 (2012)

4. Badawy, A., El-Fawal, R.: Homomorphism and Subalgebras of decomposable $M S$-algebras. J. Egypt. Math. Soc. 25, 119-124 (2017)

5. Badawy, A.: $d_{L}$-Filters of principal $M S$-algebras. J. Egypt. Math. Soc. 23, 463-469 (2015)

6. Badawy, A.: Congruences and de Morgan filters of decomposable MS-algebras. SE Asia. Bull. Math. 43, 13-25 (2019)

7. Badawy, A., Sambasiva Rao, M.: Closure ideals of $M S$-algebras. Chamchuri J. Math. 6, 31-46 (2014)

8. Badawy, A.: $\delta$-ideals in $M S$-algebras. J. Comput. Sci. Syst. Biol. 9(2), 28-32 (2016)

9. Chen, C. C., Grätzer, G.: Stone lattices II. Structure Theorems. Can. J. Math. 21, 895-903 (1969)

10. El-Assar, S., Atallah, M.: Completeness of distributive $p$-algebras. Qater Univ. Sci. Bull. 8, 29-33 (1988)

11. Mederly, P.: A characterization of complete modular p-algebras. Colloq. Math. Soci. János Bolyai. 17, 211-329 (1975). Contributions to Universal Algebra (Hungary)

12. Blyth, T. S., Varlet, J. C.: Ockham Algebras. Oxford University Press, Oxford (1994)

13. Blyth, T. S.: Lattices and ordered algebraic structures. Springer-Verlag, London (2005)

14. Grätzer, G.: Lattice theory, first concepts and distributive lattices, Lecture Notes. Freeman, San Francisco (1971)

15. Badawy, A.: Extensions of the Glivenko-type congruences on a Stone lattice. Math. Meth. Appl. Sci. 41, 5719-5732 (2018)

\section{Submit your manuscript to a SpringerOpen ${ }^{\circ}$ journal and benefit from:}

- Convenient online submission

- Rigorous peer review

- Open access: articles freely available online

- High visibility within the field

- Retaining the copyright to your article

Submit your next manuscript at $\boldsymbol{\triangleright}$ springeropen.com 